\title{
Tracheal wall thickening: An important radiologic feature of relapsing polychondritis
}

\author{
Masafumi Abe, Satoshi Yoshikawa, Takeshi Ueda \\ From Medical Doctor, Emergency and General Internal Medicine, Rakuwakai Marutamachi Hospital, Kyoto, Japan
}

$\mathrm{A}$ previously healthy 49-year-old woman presented after experiencing cough, fever, and sore throat. One month earlier, cough and sore throat had developed, which had been gradually worsening. She also reported a fever for three days. She denied auricular pain, eye redness, and nose pain. She had been treated with an antihistamine, long-acting $\beta 2$-agonist, and inhaled corticosteroid, but her symptoms had not changed. Her initial vital signs showed a temperature of $36.3^{\circ} \mathrm{C}$, a heart rate of 101 beats per minute, a blood pressure of $102 / 58 \mathrm{~mm} \mathrm{Hg}$, a respiratory rate of 12 breaths per minute, and an oxygen saturation of $98 \%$ while the patient was breathing ambient air. Physical examination revealed tenderness of the several costosternal joints without wheezing and stridor. Laboratory results showed an erythrocyte sedimentation rate of $119 \mathrm{~mm}$ per hour (reference range, 1-11). A chest radiograph showed a diffusely thickened right paratracheal stripe (Fig. 1a). She was treated with oral prednisolone at a dose of $25 \mathrm{mg}$ per day, and her symptoms were alleviated within a week. She had chondritis in two separate anatomic locations that responded to prednisolone. Therefore, a diagnosis of relapsing polychondritis (RPC) was made. A repeat chest radiograph 8 months after the initial presentation showed complete resolution of the tracheal wall thickening (Fig. 1b).

RPC is a rare autoimmune disorder in which the cartilaginous tissues are the primary targets of destruction. More than half of patients with RPC will develop respiratory symptoms such as dyspnea, cough, and stridor during the course of the disease [1]. The radiological features of RPC are focal or diffuse smooth wall thickening of the anterior and lateral tracheal walls [2] The differential diagnosis for tracheal wall thickening includes amyloidosis, sarcoidosis, ulcerative colitis, granulomatosis with polyangiitis, tracheopathia osteochondroplastica, various infections, asthma, and

\section{Access this article online}

Received - 13 January 2022

Initial Review - 21 January 2022

Accepted - 04 February 2022

DOI: $10.32677 /$ ijcr.v8i1.3328

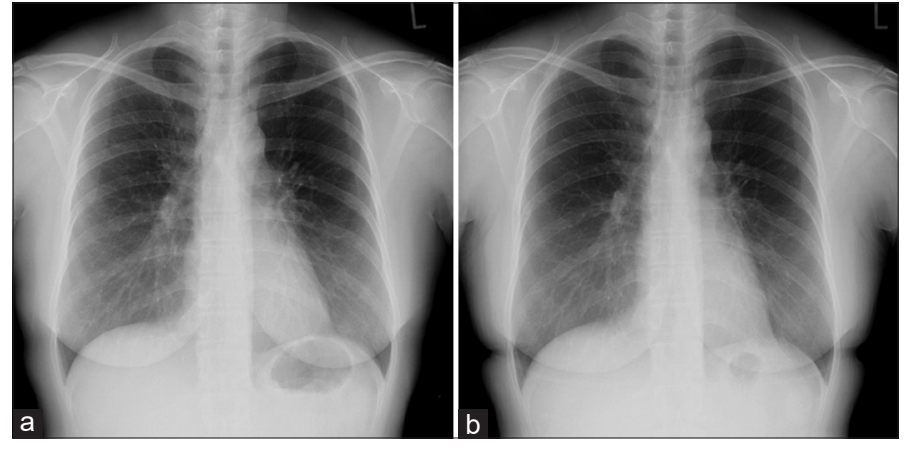

Figure 1: (a) Chest radiograph (a) on presentation showed diffusely thickened right paratracheal stripe; (b) after eight months showed complete resolution of the tracheal wall thickening

malignancies [3]. This case illustrates that a chest radiograph can show respiratory involvement of RPC.

\section{REFERENCES}

1. Kent PD, Michet CJ Jr., Luthra HS. Relapsing polychondritis. Curr Opin Rheumatol 2004;16:56-61.

2. Rafeq S, Trentham D, Ernst A. Pulmonary manifestations of relapsing polychondritis. Clin Chest Med 2010;31:513-8.

3. Marom EM, Goodman PC, McAdams HP. diffuse abnormalities of the trachea and main bronchi. AJR Am J Roentgenol 2001;176:713-7.

Funding: None; Conflicts of Interest: None Stated.

How to cite this article: Abe M, Yoshikawa S, Ueda T. Tracheal wall thickening: An important radiologic feature of relapsing polychondritis. Indian J Case Reports. 2022;8(1):22.

Correspondence to: Masafumi Abe, Division of Emergency and General Internal Medicine, Rakuwakai Marutamachi Hospital, 9-7 Jyurakumawari-Matsushitacho, Marutamachi, Nakagyo-ku, Kyoto 604-8401, Japan. E-mail: kyokui080002@ gmail.com

(C) 2022 Creative Commons Attribution-NonCommercial 4.0 International License (CC BY-NC-ND 4.0). 